\title{
Swissvasc Registry ist seit 1. Januar 2004 online verfügbar!
}

\author{
P. Wigger
}

Swissvasc Registry ist ein Projekt zur Qualitätssicherung, welches von der Schweizerischen Gesellschaft für Gefässchirurgie (SGG) und der Schweizerischen Gesellschaft für Thorax-, Herz- und Gefässchirurgie (SGTHG) getragen wird.

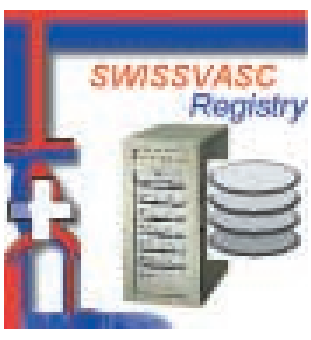

Korrespondenz:

Dr. med. Pius Wigger

Kantonsspital

Brauerstrasse 15

Postfach 834

CH-8401 Winterthur

E-Mail: p.wigger@ksw.ch

\section{Ziel}

Ziel ist es, auf nationaler Ebene in einer zentralen Datenbank möglichst viele der in der Schweiz durchgeführten gefässchirurgischen Eingriffe inklusive deren postoperativen Verlauf $\mathrm{zu}$ erfassen. Das Register dient dazu, einen gesamtschweizerischen Überblick und eine Qualitätskontrolle zu erhalten. Damit kann die Gefässchirurgie in der Schweiz zum Standard aufschliessen, der in andern europäischen Ländern (Danvasc, Swedvasc u.a.) bereits Realität ist.

\section{Organisation von Swissvasc Registry}

Das Projekt wird von der Schweizerischen Gesellschaft für Gefässchirurgie (SGG) und der Schweizerischen Gesellschaft für Thorax-, Herzund Gefässchirurgie (SGTHG) getragen. Die Präsidenten dieser beiden Gesellschaften bilden zusammen mit dem Projektleiter den Steuerungsausschuss. Der Steuerungsausschuss beschliesst Projektrahmen und Projektänderungen und ist Vertragspartner der Softwarefirma.

Abbildung 1

Organisation Swissvasc Registry.

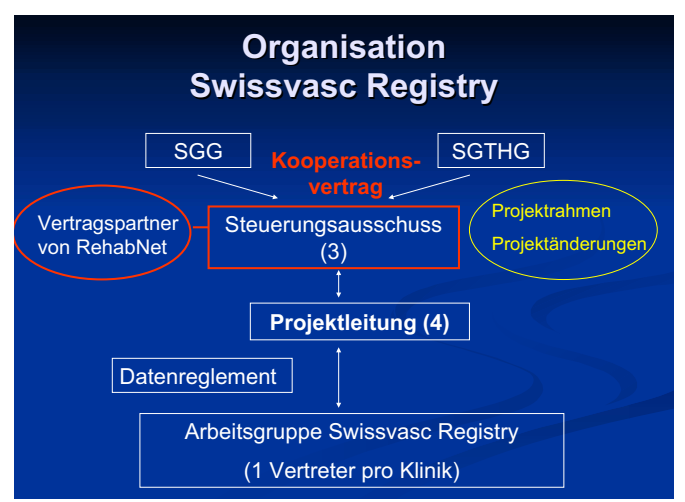

Folgende Personen sind in der Projektleitung für das Projekt verantwortlich:

- Dr. med. Pius Wigger, Leitender Arzt Gefässchirurgie, Kantonsspital Winterthur;

- Dr. Jürg Schmidli, Leiter Abteilung Gefässchirurgie, Inselspital Bern;

- PD Dr. Lorenz Gürke, Leitender Arzt Gefässchirurgie, Universitätsklinik Basel;

- Dr. Dieter Mayer, Oberarzt Abteilung für Gefässchirurgie, USZ, Zürich.

\section{Arbeitsgruppe Swissvasc Registry}

Jede teilnehmende Klinik schickt einen Vertreter in die Arbeitsgruppe.

\section{Technische Aspekte}

Die Daten werden auf einem zentralen Server der Firma Rehabnet (www.rehabnet.ch) in Zürich, anonymisiert nach den Richtlinien des eidgenössischen Datenschutzbeauftragen (Jahrgang, Geschlecht und die vom User gewählte Patientennummer), gespeichert. Die Dateneingabe erfolgt entweder direkt via Internet mit OnlinePlausibilisierung und Online-Eingabehilfen oder via scanbaren Fragebogen.

Der Zugang für die Online-Eingabe erfolgt über die Internetseite www.rehabnet.ch/SWISSVASC. Es werden ein Benutzername und ein Passwort benötigt. Die Datenbank ist mit einer Firewall geschützt.

Jede Klinik hat die Möglichkeit, alle eigenen Daten einzusehen und via Excel- bzw. SPSS-File jederzeit zu exportieren. Jede Klinik hat selbstverständlich nur Zugriff auf die eigenen Daten. Über die Patientennummer ist es der Klinik möglich, den Patienten wieder zu identifizieren.

Es wird einmal jährlich eine Auswertung der Daten des Gesamtkollektivs vorgenommen und nach Genehmigung durch die Projektleitung veröffentlicht. Anonyme Klinikvergleiche sind erst nach 2 bis 3 Jahren vorgesehen. Jede Klinik kann eine Jahresauswertung der eigenen Daten bestellen. 


\section{Was geschah bisher?}

Die ersten Anstrengungen gehen ins Jahr 1999 zurück. Dort wurde anlässlich der Mitgliederversammlung der SGG von Prof. A. Marx und Dr. D. Mayer ein nationales Register auf der Basis des nordirischen Fragebogens vorgeschlagen. Im Februar 2001 setzte der Vorstand der SGG eine Arbeitsgruppe Swissvasc Registry ein. Nach umfangreichen Vorarbeiten bezüglich Organisationsform, Datenbankstruktur, Finanzierung und Softwarepartner erfolgte der eigentliche Startschuss im Juni 2002 durch den Beschluss der GV der SGTHG, Swissvasc Registry als nationale Gefässdatenbank einzuführen. Zwei Monate später unterzeichneten zwölf Kliniken der Schweiz einen «Letter of Intent», in dem sie sich zur Teilnahme und Mitfinanzierung der laufenden Kosten verpflichteten. Alle Universitätskliniken waren mit dabei. Ende 2002 wurde der Kooperationsvertrag zwischen der Steuerungsgruppe und der Firma Rehabnet unterzeichnet. Seit April 2003 testeten sieben Pilotkliniken den Fragebogen und die Datenbank. Die Erfahrungen wurden Ende 2003 umgesetzt und führten zum nun vorliegenden Fragebogen.
Im Dezember 2003 wurde an alle Chefärzte der chirurgischen Kliniken ein Informationsschreiben sowie ein Anmeldebogen für die Teilnahme am Swissvasc Register geschickt. Eine ganze Reihe von Kliniken hat sich erfreulicherweise bereits zur Teilnahme angemeldet.

\section{Wer macht mit?}

Beim Projektstart haben sich alle Universitätskliniken und sieben Kantonsspitäler in einem «Letter of Intent» verpflichtet, an diesem Projekt teilzunehmen und ihre Daten einzugeben. Für die Ausbildungskliniken mit Schwerpunkt Gefässchirurgie ist die Teilnahme obligatorisch. Alle übrigen Kliniken können auf freiwilliger Basis mitmachen.

\section{Kosten?}

Die Kosten setzen sich aus den jährlichen Fixkosten für die gesamte Datenbank sowie den Kliniklizenzgebühren zusammen. Sie werden nach Anzahl dokumentierter Patienten berechnet. Kleinere Kliniken dürfen mit Teilnahmekosten von deutlich unter Fr. 1000.- pro Jahr rechnen. 